\title{
MENTAL RETARDATION, PIGMENTATION OF THE SKIN, XANTHOMATOSIS AND LEUKAEMIA
}

\author{
BY \\ J. L. EMERY, J. LORBER and J. D. SHEWARD \\ From the Sheffield Children's Hospital and the University of Sheffield
}

(RECEIVED FOR PUBliCATION JULY 20, 1955)

The syndrome to be described, namely skin pigmentation followed by xanthomatosis and subsequently myeloid leukaemia, in a mentally retarded child has apparently not been described before.

\section{Case History}

G.S., a boy, the third child in a family, was born at term by normal delivery at the Jessop Hospital for Women, Sheffield, on February 9, 1951, after an uncomplicated pregnancy. He weighed $5 \mathrm{lb}$. $12 \mathrm{oz}$. No abnormalities were noted at birth, and progress during the neonatal period was uneventful. There was no family history of lipoid storage disease. As the mother suffered from active pulmonary tuberculosis, the infant was separated from her and was vaccinated with B.C.G. This procedure was followed by Mantoux conversion. He was examined at intervals in the tuberculosis contact clinic of the Sheffield Children's Hospital. At the age of 14 weeks he weighed $13 \mathrm{lb}$. $3 \mathrm{oz}$. He was well, could smile, could hold objects put into his hand, and was able to lift his head momentarily from the supine position. At the age of 46 weeks he weighed $17 \mathrm{lb} .6 \mathrm{oz}$. He was quite well, but was considered to be retarded at this stage. He refused to have solid foods. He made no attempt to pull himself into the sitting position, although he could sit without support when he was put there. He would say syllables; he would only occasionally laugh; he had no interest in his toys and he only very occasionally transferred an object from one hand to the other. By now, he was found to have café-au-lait spots and he had an enlarged spleen.

At 1 year of age he weighed $17 \frac{1}{2} \mathrm{lb}$. He showed no improvement in his mental condition. He was unable to get into the sitting position. He could not crawl and made no attempt to stand. He now rapidly transferred objects from one hand to the other. He could not say a single word. Professor Illingworth considered at this time that he was severely backward with a developmental quotient of approximately 75 . The physical signs had not altered. He had no xanthomata.

At 14 months of age he was admitted for investigation. He had suffered from a cough for three weeks before his admission but was not acutely ill. He weighed
$16 \mathrm{lb} .1 \mathrm{oz}$. There was a nasal discharge and scattered rales and rhonchi were heard over both lungs. The spleen was enlarged and firm, reaching three fingerbreadths below the costal margin. The liver edge was palpable an inch below the costal margin and small, discrete enlarged lymph nodes were felt in the axillae, groins and neck. Scattered café-au-lait patches of pigmentation were seen in the skin, but no other cutaneous abnormalities were found. No other abnormal physical signs were detected.

Haematological findings were as follows: Haemoglobin, 10 g. $\%$; white cell count, 17,400/c.mm. (polymorphs $38 \%$, lymphocytes $48 \%$, eosinophils $2 \%$, monocytes $12 \%$ ). No primitive cell forms were seen in the peripheral blood. The bone marrow smear showed some depression in erythropoiesis, but no evidence of leukaemia. The cerebrospinal fluid and urine showed no abnormalities. The blood and C.S.F. Wassermann reactions were negative.

After discharge home he remained symptom free, though backwardness persisted. He was about 2 years old when he first walked without assistance, and at this time his speech was still limited to monosyllables.

At the time of his final admission to hospital at 3 years of age, the boy had been ill for about a month. He was not eating well and was noticeably thinner. A loose productive cough had developed with fever, lassitude and general malaise. On admission he looked pale, thin and acutely ill. He had a loose, distressing cough with a mucopurulent nasal discharge and fever. The percussion note was impaired at the apex of the right lung, and there were generalized rales and rhonchi. The abdomen was distended and ascitic. The scrotum and ankles were oedematous. The liver was firm, enlarged and palpable 2 in. below the costal margin. The spleen was palpable 3 in. below the costal margin, and the impression of its lower margin could be seen against the lower abdominal wall. Discrete, enlarged, firm lymph nodes were palpable in the cervical axillary and inguinal regions. Scattered café-au-lait patches were still present in large numbers, and some of them were very large. A new feature was xanthomata as bright yellow papules, 0.5 to $1.0 \mathrm{~cm}$. in diameter, sparsely scattered on the chest and abdomen, but more heavily 
distributed on the scalp. A typical xanthomatous plaque was also seen on one conjunctival surface. No petechiae were visible at this time. No abnormal signs were found in the central nervous system. The optic fundi were normal. There was no evidence of cardiovascular disease. Mental retardation was still evident, though its exact degree could not be determined because he was now very ill.

During this admission full blood investigations were made and the following results obtained: Haemoglobin, $8 \cdot 3$ g. $\%$; red blood cells, $2,900,000 /$ c.mm.; nucleated red cells, one per 300 leucocytes; platelets, $21,000 / \mathrm{c} . \mathrm{mm}$.; total white cell count, $266,000 /$ c.mm. (polymorphs $57 \%$, monocytes $22 \%$, basophils nil, myelocytes $9 \%$, eosinophils nil, lymphocytes $10 \%$, primitive cells $2 \%$ ). The bleeding time was 8 minutes 45 seconds (Duke's method). The clotting time was 6 minutes (capillary method). Bone marrow smears showed abnormal forms in all the blood elements. The picture was that of chronic myeloid leukaemia. Tubercle bacilli were not demonstrated by appropriate techniques.

An xanthomatous plaque was removed under local anaesthesia and the histological appearances confirmed the presence of xanthoma cells with no evidence of neoplastic infiltration.

Biochemical investigations gave the following results: Total serum lipoids, $483 \mathrm{mg}$. \%; serum cholesterol, $59 \mathrm{mg}$. \%; thymol turbidity, 12 units; zinc sulphate test, 60 units; serum albumin, $2 \cdot 3 \mathrm{~g}$. \%, and serum globulin, $2 \cdot 4$ g. $\%$.

Serum electrophoresis demonstrated increased gamma globulin levels.

Apart from a trace of albumin the urine was normal.

A radiograph of the chest showed a dense opacity in the right upper zone with a convex lower margin (Fig. 1). There was calcification in the axillary lymph nodes on both sides, as a result of the B.C.G. inoculation. The Mantoux reaction, 1 in 1,000 , was positive. No significant abnormalities were seen in the radiographs of the skull and long bones.

The respiratory symptoms and chest radiograph led

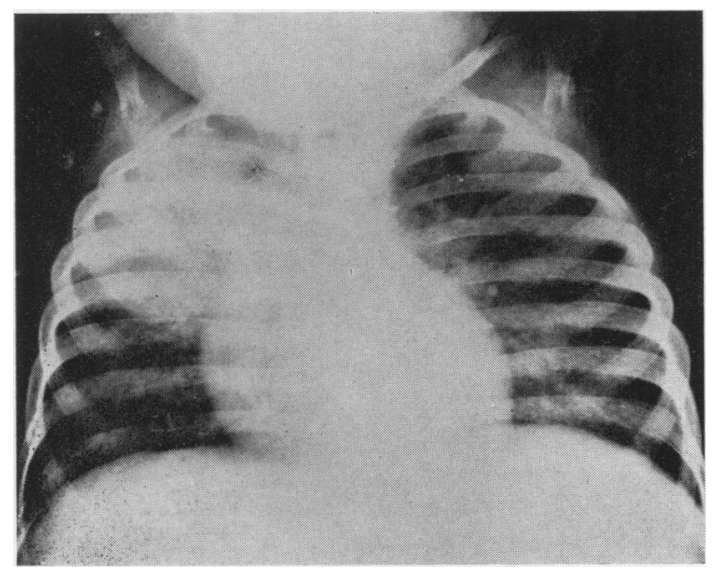

FIG. 1.-Radiograph of chest 10 weeks before death.

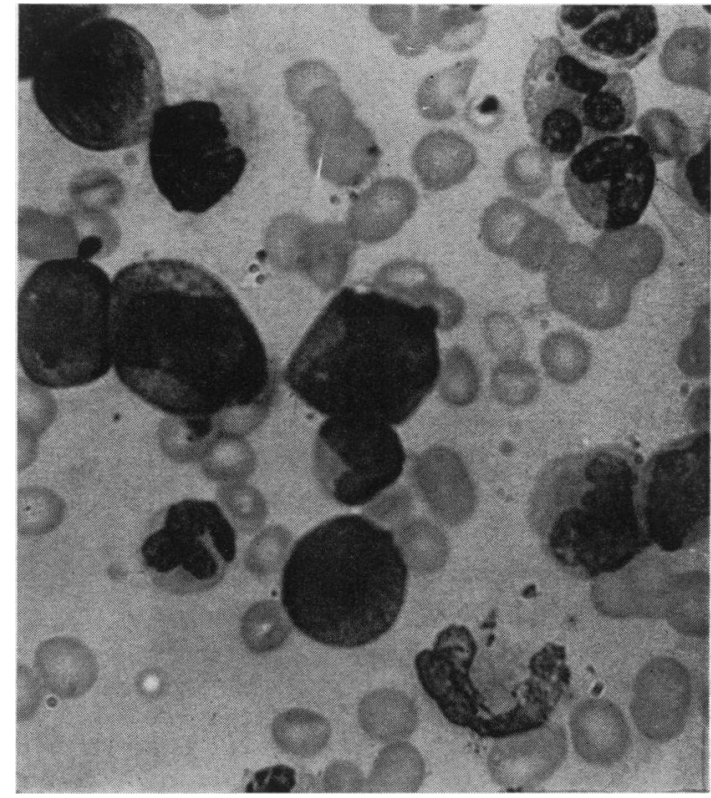

FIG. 2.-Peripheral blood smear showing the leukaemic blood picture.

to a diagnosis of an unresolved right upper lobe pneumonia. Following large doses of penicillin, there was some improvement in the child's respiratory distress with a fall in temperature, but his general condition worsened, with increasing pallor, oedema and ascites. Further radiographs of the chest showed no improvement. Later there was a further increase in the respiratory distress accompanied by high fever. Generalized petechial haemorrhages appeared in the skin. His general condition deteriorated and did not respond to courses of soluble penicillin and aureomycin. Further haematological examinations revealed an increasing degree of anaemia, the haemoglobin steadily falling to $4 \cdot 2 \mathrm{~g}$. \%, persistence of the leucocytosis with an eventual rise to $336,000 /$ c.mm. (Fig. 2) and a progressive thrombocytopenia. Urethane, $0 \cdot 125 \mathrm{~g}$., was given six hourly for several days without effect on the white cell count. He ran a continuous irregular high fever throughout the latter part of his illness, and his condition slowly deteriorated until he died at the age of 3 years and 3 months, 10 weeks after his admission.

Necropsy. The body was that of a wasted male child with no anatomical deformities. There was oedema of the feet, lower parts of the legs, sacrum, forearms and hands. The skin showed two distinct lesions. There were large café-au-lait patches, particularly on the trunk, of approximately $2 \mathrm{~cm}$. in diameter (Fig. 3), and there was a much larger number of discrete, slightly raised xanthomatous areas-particularly prominent over the scalp and face, and varying from 2 to $8 \mathrm{~mm}$. in diameter.

The peritoneal and pericardial cavities contained sero- 


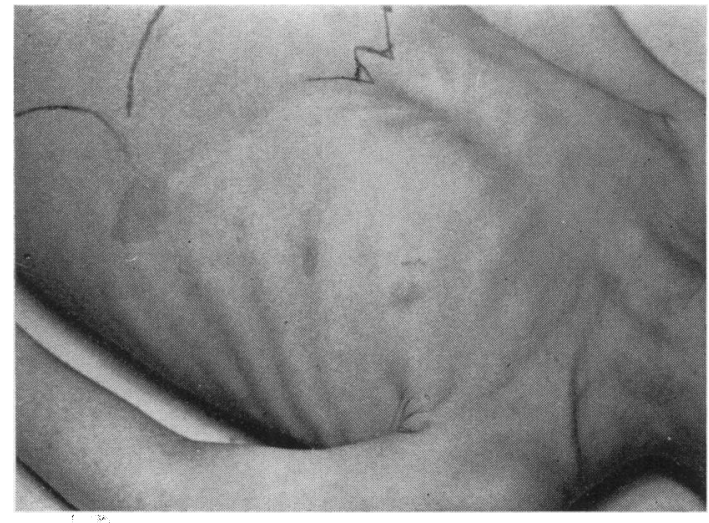

FIG. 3. - Necropsy view of the upper trunk showing the areas of skin pigmentation and the size of the liver and spleen.

sanguinous fluid, and within the serous membrane were a number of almost circular xanthomata (Fig. 4).

The left pleural cavity had firm adhesions and the right was almost completely obliterated except for the midlateral third where there was a chronic empyema extending into the intralobular fissure. The respiratory tract contained mucopus and in the lungs there were many areas of consolidation.

The heart muscle was very pale, and xanthomatous areas were present in the connective tissue around the coronary arteries. Similar areas were present in the liver and kidneys.

The intestinal tract showed no abnormality other than a large number of small haemorrhages in and from the mucosa.

The liver was enlarged and weighed $855 \mathrm{~g}$. (normal 400-500 g.). It was pale, with yellow xanthomatous areas around many vessels. The spleen was large and weighed $296 \mathrm{~g}$. (normal 35-40 g.). It was very firm and deep red. The kidneys were pale and not grossly enlarged and weighed $132 \mathrm{~g}$. (normal 90-120 g.). The other viscera showed no gross changes, and the endocrine glands appeared normal. The brain was of normal weight and showed no gross abnormalities, no xanthomata being seen around the superficial vessels.

The lymph nodes in the axilla associated with the B.C.G. inoculation were small and calcified. Throughout the rest of the body the nodes were enlarged, particularly in the mediastinum and mesentery. None showed caseation or necrosis.

Histology. The general architecture of the lymph nodes, the spleen and the bone marrow was almost entirely replaced by diffuse masses of primitive cells. Similar types of cells were present in large infiltrating masses in the kidneys, intestinal mucosa, liver, testes and in a lesser degree in all of the other organs examined with the exception of the thymus and the brain.

The xanthomatous areas were entirely devoid of the leukaemic infiltration, and contained both neutral fats and doubly refractile material, as in the biopsy specimen.
The lung contained many acute abscesses as well as evidence of long-standing infection, and there was considerable alveolar oedema.

An extensive search was made for intranuclear inclusion bodies in the liver, kidney and other organs, but none were found. No abnormality was recognized in the structure of the brain in preparations by routine methods.

\section{Discussion}

This child presented when he was a year old with mental retardation, splenomegaly, lymphadenopathy and patchy cutaneous pigmentation. For the next two years his general health was fair, although his mental retardation and splenomegaly persisted. The appearance of scattered cutaneous xanthoma was noted when his health rapidly deteriorated at the age of 3 years. He had extreme leucocytosis and the bone marrow appearances were those of a chronic myeloid leukaemia. Necropsy revealed xanthomatous lesions in the viscera, leukaemia and gross pulmonary infection.

Skin xanthomata occur in a number of diseases in childhood (Crocker, 1951; Freud, 1951; Thannhauser, 1950) and are usually clinically separated into two broad groups, based on the serum cholesterol and fat levels. The group in which the serum cholesterol level is usually normal, as in our case, includes a benign form of cutaneous xanthoma, various chronic diseases especially the 'eosinophilic granuloma syndromes' (Thannhauser, 1950), leukaemia and von Recklinghausen's neurofibromatosis (Crocker, 1951).

Most cases with visceral juvenile xanthoma had a fatal outcome (Thelander, 1949) but Thannhauser (1950) and Crocker (1951) each recorded a child whose general health remained good.

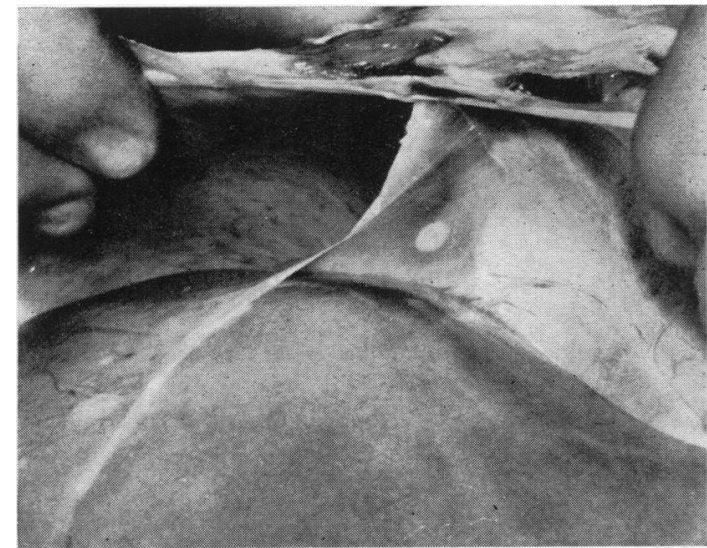

Fig. 4.-Photograph of the upper surface of the liver falciform ligament and diaphragm. There is a round xanthomatous plaque in the ligament and another on the upper surface of the liver. 
Mental deterioration occurs in the infantile form of Gaucher's disease and Niemann-Pick's disease, but was not described in the cases of xanthomatosis of Thannhauser (1950) and Crocker (1951). Thelander (1949) reported a child with xanthomata, hepatosplenomegaly and mental retardation, who died after recurrent periods of ill health lasting 20 months. The bone marrow at no time showed evidence of leukaemia and the brain appeared normal as in our case.

The only previous description of a child that corresponds closely to the case reported here is that which Freud and his colleagues reported in a series of four papers (Freud, 1951 and 1952; Freud, Plachta, Speer and Luhby, 1954; Freud and Plachta, 1954). Their child presented with xanthomatous skin lesions. Twenty-five months later the child developed a generalized reticulo-endotheliosis and later developed leukaemia. At necropsy numerous inclusion bodies were found, particularly in the liver. The present case differs principally in the final point, in that after a prolonged search we were unable to find intranuclear inclusions. It was upon the finding of the intranuclear inclusions that it was suggested that the condition may have had an infective origin. If these two cases are in fact similar it would suggest that the presence of intranuclear inclusions in the earlier reported case was fortuitous, and that the disease syndrome is a general non-specific neoplastic disorder of the reticuloendothelial system.

\section{Summary}

A case is reported of a child suffering from a systemic reticulo-endotheliosis, characterized by mental retardation, hepatosplenomegaly, lymphadenopathy and cutaneous xanthoma, who later developed chronic myeloid leukaemia.

We are indebted to Professor R. S. Illingworth for his criticism of this paper.

\section{REFERENCES}

Crocker, A. C. (1951). Pediatrics, 8, 573.

Freud, P. (1951). J. Pediat., 38, 744.

(1952). J. Mt Sinai Hosp., 19, 243.

(1952). J. Mt Sinai Hosp., 19, 243.

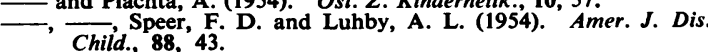

Thannhauser, S. J. (1950). Lipidoses. (Specifically case 43, p. 410.) New York.

Thelander, H. E. (1949). J. Pediat., 34, 490. 